\title{
DIFFERENTIAL GAME WITH A LIFELINE FOR THE INERTIAL MOVEMENTS OF PLAYERS
}

\author{
Bahrom T. Samatov ${ }^{\dagger}, \quad$ Ulmasjon B. Soyibboev ${ }^{\dagger \dagger}$ \\ Namangan State University, \\ 316 Uychi Str., Namangan, 116019, Uzbekistan \\ †samatov57@inbox.ru, ††ulmasjonsoyibboev@gmail.com
}

\begin{abstract}
In this paper, we study the well-known problem of Isaacs called the "Lifeline" game when movements of players occur by acceleration vectors, that is, by inertia in Euclidean space. To solve this problem, we investigate the dynamics of the attainability domain of an evader through finding solvability conditions of the pursuit-evasion problems in favor of a pursuer or an evader. Here a pursuit problem is solved by a parallel pursuit strategy. To solve an evasion problem, we propose a strategy for the evader and show that the evasion is possible from given initial positions of players. Note that this work develops and continues studies of Isaacs, Petrosjan, Pshenichnii, Azamov, and others performed for the case of players' movements without inertia.
\end{abstract}

Keywords: Differential game, Pursuit, Evasion, Acceleration, Strategy, Attainability domain, Lifeline.

\section{Introduction}

Differential game theory deals with conflict problems in systems expressed by differential equations. As a result of developing Pontryagin's maximum principle, it became apparent that there was a link between optimal control theory and differential games. Actually, problems of differential games describe a generalization of optimal control problems in cases where more than one player is involved.

The study of differential games was initiated by American mathematician R. Isaacs. His research was published in the form of a monograph [20] in 1965, in which a great number of examples were considered, and theoretical questions were only affected. Differential games have been one of the basic research fields since 1960, and their fundamental results were gained by Pontryagin [29], Krasovskii [23], Bercovitz [5], Dar'in and Kurzhanskii [9], Elliot and Kalton [10], Isaacs [20], Fleming [11], Friedman [12], Hajek [14], Ho, Bryson and Baron [15], Petrosjan [28], Pshenichnii [30, 31], Subbotin [40, 41], Ushakov [42], Chikrii [8], and others.

The monograph of Isaacs [20] includes certain game problems that were discussed in detail and put forward for further study. One of these problems is called the "Lifeline" problem, which was initially formulated and solved for certain special cases [20, Problem 9.5.1]. A simplified analytical solution to this problem in the half-plane was proposed by Isaacs in [20]. For the case when controls of both players are subjected to geometric constraints, this game was rather thoroughly considered by Petrosjan [28] based on approximating measurable controls with the most efficient piecewise constant controls that realize the parallel convergence strategy. Later, this approach to control in differential pursuit games was termed the П-strategy. The strategy proposed in [28], for a simple pursuit game with geometric constraints, became the starting point for the development of the pursuit method in games with multiple pursuers $[6,13,39]$. In 1986, a simplified analytical solution of the "Lifeline" problem was suggested by A. Azamov [3]. Later on, for the cases when controls of both players are subjected to integral, Grönwall or mixed constraints, this game was investigated in the works of Samatov [4, 32-37]. 
Based on the fundamental approaches in the theory of differential games developed by Pontryagin [29] and Krasovskii [23], a differential game is considered as a control problem from the point of view of either pursuer or evader. According to this view, the game reduces to either pursuit (convergence) problem or evasion (escape) problem. The main technique for solving the pursuit and evasion problems is constructing optimal strategies of players and defining the value of the game. The works $[16-19,21]$ are devoted to studying differential games of simple motion and by optimal strategies of players, it was proved that the value of the game exists.

In $[24,25]$, the classic time-optimal differential games with a lifeline were investigated. The first player seeks to lead the system to a given closed terminal set with a smooth boundary, and the second player strives to guide the same system to another given set whose boundary is also smooth and is also called a lifeline. To solve this problem, the authors adhered to the formalization of positional differential games proposed by N.N. Krasovskii and A.I. Subbotin.

In the present paper, we discuss the pursuit-evasion problems and the "Lifeline" game for the inertial movements of players. We impose geometric constraints on controls of the players. In order to solve a pursuit problem, we suggest the П-strategy for the pursuer and prove that this is an optimal strategy. After that, necessary and sufficient condition of pursuit is originated and optimal pursuit time (guaranteed pursuit time) is determined. To solve an evasion problem, we propose a strategy for the evader and show that the evasion is possible from given initial positions of the players. Here, any closed set given in the space is considered as a lifeline. In this case, the first player (a pursuer) aims to coincide with the second player (an evader) as quickly as possible and, by this occurrence, a trajectory of the evader shouldn't intersect the lifeline. The aim of the evader is to reach the lifeline by the time of the coincidence or is not to encounter the pursuer during the game. To solve the "Lifeline" problem, conditions of monotone embedding in respect to time for an attainability domain are given. In this paper, the statement and solution method of a differential game with a lifeline differ significantly from those from the works [24, 25]. Results of the paper rely on the works $[1,7,22,26-29,42,43]$ and adjoin the works [2-4, 20, 38, 41].

\section{Statement of problem}

Assume that in the space $\mathbb{R}^{n}$ a controlled object $P$, called a pursuer, chases another object $E$, called an evader. Denote by $x$ a state of the pursuer and by $y$ that of the evader in $\mathbb{R}^{n}$.

Let the motion dynamics of the players be generated by the following differential equations and initial conditions respectively:

$$
\begin{array}{lll}
P: \ddot{x}=u, & x(0)=x_{0}, & \dot{x}(0)=x_{1}, \\
E: \ddot{y}=v, & y(0)=y_{0}, & \dot{y}(0)=y_{1},
\end{array}
$$

where $x, y, x_{0}, y_{0}, x_{1}, y_{1}, u, v \in \mathbb{R}^{n}, n \geq 2 ; x_{0}$ and $y_{0}$ are initial states of the players, and $x_{1}$ and $y_{1}$ are their initial velocity vectors, respectively. We suppose that $x_{0} \neq y_{0}$ and $x_{1}=y_{1}$.

Here the temporal variation of $u$ must be a measurable function $u(\cdot):[0, \infty) \rightarrow \mathbb{R}^{n}$, and we impose a geometrical constraint on this vector-function (briefly, $G$-constraint) in the form

$$
|u(t)| \leq \alpha \quad \text { almost everywhere } \quad t \geq 0
$$

where $\alpha$ is a given positive parametric number, which means the maximal acceleration value of the pursuer.

In a similar way, the temporal variation of $v$ must be a measurable function $v(\cdot):[0, \infty) \rightarrow \mathbb{R}^{n}$, and we impose on this vector-function a $G$-constraint in the form

$$
|v(t)| \leq \beta, \quad \text { almost everywhere } \quad t \geq 0,
$$


where $\beta$ is a given non-negative parametric number, which means the maximal acceleration value of the evader.

The control parameters of the players are the acceleration vectors $u$ and $v$, which depend on time $t \geq 0$. We denote by $U$ and $V$ the set of all control parameters $u$ and $v$ satisfying conditions (2.3) and (2.4), respectively.

Definition 1. We call the measurable function $u(\cdot)(v(\cdot))$ that satisfies the condition (2.3) (the condition (2.4)) an admissible control of the pursuer (the evader) of the class $U$ (the class $V$ ), where the pair of classes of admissible controls introduced $(U, V)$ defines a differential game.

By means of the equations (2.1) and (2.2), each triplet $\left(x_{0}, x_{1}, u(\cdot)\right)$ and $\left(y_{0}, y_{1}, v(\cdot)\right)$ generates the trajectories of motion

$$
\begin{aligned}
& x(t)=x_{0}+x_{1} t+\int_{0}^{t}(t-s) u(s) d s, \\
& y(t)=y_{0}+y_{1} t+\int_{0}^{t}(t-s) v(s) d s
\end{aligned}
$$

of the pursuer and evader respectively.

Suppose that a closed subset $M$ called a lifeline is given in the space $\mathbb{R}^{n}$. The main goal of a pursuer $P$ is to catch an evader $E$, that is, to achieve the equality $x\left(t^{*}\right)=y\left(t^{*}\right)$ at some time $t^{*}$, $t^{*}>0$, while the evader remains in the zone $\mathbb{R}^{n} \backslash M$. The goal of the evader is to reach the zone $M$ before being caught by the pursuer or to maintain the relation $x(t) \neq y(t)$ for all $t, t \geq 0$. We should note that $M$ does not restrict the motion of the pursuer. Further, it is assumed that the initial states $x_{0}$ and $y_{0}$ are given under the conditions $x_{0} \neq y_{0}$ and $y_{0} \notin M$.

It is known that control functions depending only on the time parameter $t, t \geq 0$, are insufficient for the pursuer to solve the pursuit problem, and suitable types of control must be strategies. There are several methods for defining such a concept. Below we will give some concepts to define.

First, we introduce the following notation:

$$
z(t)=x(t)-y(t), \quad z_{0}=x_{0}-y_{0}, \quad \dot{z}(0)=x_{1}-y_{1} .
$$

Definition 2. A function $\boldsymbol{u}: \mathbb{R}^{n} \times S_{\beta} \rightarrow S_{\alpha}$ is called a strategy of the pursuer if the following conditions are valid.

(a) $\boldsymbol{u}\left(z_{0}, v\right)$ is a Borel measurable function in $v, v \in V$.

(b) Admissibility: The inclusion $\boldsymbol{u}\left(z_{0}, v(\cdot)\right) \in U$ holds for each $v(\cdot) \in V$ on some time interval $[0, t]$. In this case, the function $\boldsymbol{u}\left(z_{0}, v(\cdot)\right)$ is called a realization of the strategy $\boldsymbol{u}\left(z_{0}, v\right)$.

(c) Volterra property: If $v_{1}(s)=v_{2}(s)$ almost everywhere on $[0, t]$ for every $v_{1}(\cdot), v_{2}(\cdot) \in V$, and $t, t \geq 0$, then $u_{1}(s)=u_{2}(s)$ almost everywhere on $[0, t]$, where $u_{i}(\cdot)=\boldsymbol{u}\left(z_{0}, v_{i}(\cdot)\right), i=1,2$; $S_{\alpha}$ and $S_{\beta}$ are the balls with radii $\alpha$ and $\beta$, respectively, centered at the origin of the space $\mathbb{R}^{n}$.

Definition 3. A strategy $\boldsymbol{u}=\boldsymbol{u}\left(z_{0}, v\right)$ is called a parallel pursuit strategy, or П-strategy if, for any $v(\cdot) \in V$, a solution $z(t)$ of the Cauchy problem

$$
\ddot{z}=\boldsymbol{u}\left(z_{0}, v(t)\right)-v(t), \quad z(0)=z_{0}, \quad \dot{z}(0)=0
$$


can be expressed as

$$
z(t)=z_{0} \Lambda(t, v(\cdot)), \quad \Lambda(0, v(\cdot))=1,
$$

where $\Lambda(t, v(\cdot))$ is a scalar function of $t, t \geq 0$. Usually, this function is called a convergence function in the pursuit problem.

Definition 4. In the pursuit problem, it is said that a $\Pi$-strategy guarantees catching an evader on the time interval $\left[0, t_{g}\right]$ if, for every $v(\cdot) \in V$,

(a) there exists some time $t^{*}, t^{*} \in\left[0, t_{g}\right]$ that generates the equality $z\left(t^{*}\right)=0$;

(b) the inclusion $\boldsymbol{u}\left(z_{0}, v(\cdot)\right) \in U$ is satisfied on the time interval $\left[0, t^{*}\right]$.

Here, the number $t_{g}$ is called a guaranteed pursuit (or capture) time.

Definition 5. We call the function $\boldsymbol{v}^{*}: \mathbb{R}_{+} \rightarrow \mathbb{R}^{n}$ a strategy of the evader if $\boldsymbol{v}^{*}(t)$ is a Lebesgue measurable function in $t$.

Now we will consider the game $(U, V)$ from the standpoint of an evader.

Definition 6. In the evasion problem, it is said that a control $\boldsymbol{v}^{*}(\cdot) \in V$ guarantees escaping if, for any $u(\cdot) \in U$, a solution $z(t)$ of the Cauchy problem

$$
\ddot{z}=u(t)-\boldsymbol{v}^{*}(t), \quad z(0)=z_{0}, \quad \dot{z}(0)=0
$$

is nonzero, that is, $z(t) \neq 0$ for all $t \geq 0$.

This paper is dedicated to solving the following problems when the controls of the players are subject to constraints (2.3) and (2.4), respectively.

Problem 1. Pursuit problem: Construct a $\Pi$-strategy of the pursuer and find the guaranteed capture time in the game $(U, V)$.

Problem 2. Evasion problem: Construct a strategy of the evader and evaluate how to vary distance between the players.

Problem 3. Solve the "Lifeline" game.

\section{A solution of the pursuit problem}

In a great number of mathematical problems with parameters, an interesting property of the final analytic results is their explicit dependence on these parameters, which are regarded as constants in the solution. However, these parameters can help to determine feasibility conditions for these problems. In this section, we are going to present necessary and sufficient feasibility conditions for the pursuit problem in the game $(U, V)$.

If the pursuer and evader choose admissible controls $u(\cdot) \in U$ and $v(\cdot) \in V$, respectively, then, depending on equation (2.7), we obtain the solution

$$
z(t)=z_{0}+\int_{0}^{t}(t-s)(u(s)-v(s)) d s .
$$

In the new notation introduced, the goal of the pursuer is now to fulfill the equality $z\left(t^{*}\right)=0$ at some time $t^{*}, t^{*}>0$. As for evader's goal, it is to maintain the relation $z(t) \neq 0$ for all $t \geq 0$. 
For the pursuer, it is not enough to achieve his goal only by program strategies, i.e., admissible controls depending only on time $t$. Therefore, similarly to [4], in the case under consideration, the strategy of the pursuer can also be determined depending only on the current states of the acceleration function $v(t), t \geq 0$, and given constants $z_{0}$ and $\alpha$.

For solving the pursuit problem, suppose that at the current time $t$, the pursuer is aware of the initial parameters $x_{0}, y_{0}, x_{1}, y_{1}$, and the constants $\alpha, \beta$, the current time $t$, and the value of evader's control $v(t)$.

Definition 7. Assume that $\alpha \geq \beta$. Then, in the game $(U, V)$, we call the function

$$
\boldsymbol{u}\left(z_{0}, v\right)=v-\lambda\left(z_{0}, v\right) \xi_{0},
$$

a parallel pursuit strategy (briefly, П-strategy) of the pursuer, where

$$
\lambda\left(z_{0}, v\right)=\left\langle v, \xi_{0}\right\rangle+\sqrt{\left\langle v, \xi_{0}\right\rangle^{2}+\alpha^{2}-|v|^{2}}, \quad \xi_{0}=z_{0} /\left|z_{0}\right|,
$$

and $\left\langle v, \xi_{0}\right\rangle$ is the scalar product of the vectors $v$ and $\xi_{0}$ in $\mathbb{R}^{n}$. The function $\lambda\left(z_{0}, v\right)$ is usually called a resolving function.

Now we will indicate some important features for the strategy (3.2) and the resolving function (3.3).

Lemma 1. The strategy (3.2) is defined and continuous for all $v \in S_{\beta}$, and the equality $\left|\boldsymbol{u}\left(z_{0}, v\right)\right|=\alpha$ holds during the pursuit game.

Lemma 2. The resolving function (3.3) is defined, continuous, and non-negative for all $v \in S_{\beta}$, and this function is bounded as follows:

$$
\alpha-\beta \leq \lambda\left(z_{0}, v\right) \leq \alpha+\beta
$$

Definition 8. If $\alpha>\beta$, then the scalar function

$$
\Lambda(t, v(\cdot))=1-\frac{1}{\left|z_{0}\right|} \int_{0}^{t}(t-s) \lambda\left(z_{0}, v(s)\right) d s
$$

is called a convergence function of the players in the game $(U, V)$.

Lemma 3. Let $\alpha>\beta$. Then

(a) for all $v(\cdot) \in V$, the function (3.4) is monotone decreasing with respect to $t, t \geq 0$;

(b) the function (3.4) is bounded for all $t \in\left[0, t_{g}\right]$ as follows:

$$
\Lambda_{1}(t) \leq \Lambda(t, v(\cdot)) \leq \Lambda_{2}(t),
$$

where

$$
\Lambda_{1}(t)=1-\frac{t^{2}}{2\left|z_{0}\right|}(\alpha+\beta), \quad \Lambda_{2}(t)=1-\frac{t^{2}}{2\left|z_{0}\right|}(\alpha-\beta) .
$$


P r o o f. (a) According to Lemma 2, it follows that

$$
\frac{d \Lambda(t, v(\cdot))}{d t}=-\frac{1}{\left|z_{0}\right|} \int_{0}^{t} \lambda\left(z_{0}, v(s)\right) d s \leq-\frac{t}{\left|z_{0}\right|}(\alpha-\beta)<0 .
$$

(b) Relying on the minimum lemma in the elementary optimal control problem [1, p. 360], we get the following estimation:

$$
\Lambda(t, v(\cdot)) \leq 1-\frac{1}{\left|z_{0}\right|} \min _{v(\cdot) \in V} \int_{0}^{t}(t-s) \lambda\left(z_{0}, v(s)\right) d s \leq 1-\frac{t^{2}}{2\left|z_{0}\right|} \min _{|v| \leq \beta} \lambda\left(z_{0}, v\right)=\Lambda_{2}(t) .
$$

On the other hand, by Lemma 2, we have

$$
\Lambda_{1}(t)=1-\frac{t^{2}}{2\left|z_{0}\right|} \max _{|v| \leq \beta} \lambda\left(z_{0}, v\right)=1-\frac{1}{\left|z_{0}\right|} \max _{v(\cdot) \in V} \int_{0}^{t}(t-s) \lambda\left(z_{0}, v(s)\right) d s \leq \Lambda(t, v(\cdot)) .
$$

This completes the proof.

Theorem 1. Let $\alpha>\beta$ in the game $(U, V)$. Then the $\Pi$-strategy (3.2) guarantees catching the evader on the time interval $\left[0, t_{g}\right]$, where $t_{g}=\sqrt{2\left|z_{0}\right| /(\alpha-\beta)}$.

$\mathrm{P}$ r o o f. Assume that the evader chooses some control $v(\cdot) \in V$ while the pursuer implements the П-strategy (3.2). Then, by (3.1), we obtain the function

$$
z(t)=z_{0}+\int_{0}^{t}(t-s) \lambda\left(z_{0}, v(s)\right) \xi_{0} d s
$$

which can be written as follows:

$$
z(t)=z_{0} \Lambda(t, v(\cdot)),
$$

where $\Lambda(t, v(\cdot))$ is the players convergence function of the form (3.4). Taking into account the right-hand side of (3.5), we conclude that the function $\Lambda_{2}(t)$ is equal to zero at $t=t_{g}$. Therefore, there exists some $t^{*} \in\left[0, t_{g}\right]$ such that $\Lambda\left(t^{*}, v(\cdot)\right)=0$, and this (see (3.6)) results in $z\left(t^{*}\right)=0$. Theorem 1 is proved.

\section{A solution of the evasion problem}

In this section, we will suggest an admissible strategy for the evader, which guarantees escaping in the evasion problem. Using this strategy, we will prove that the strategy (3.2) is an optimal pursuit strategy and $t_{g}$ is an optimal pursuit time.

Definition 9. We call the control function

$$
\boldsymbol{v}^{*}(t)=-\beta \xi_{0}
$$

a strategy of the evader in the game $(U, V)$.

Definition 10. It is said that the strategy $\boldsymbol{v}^{*}(t)$ guarantees escaping on the time interval $\left[0, t_{g}\right)$ if for any control function of the pursuer $u(\cdot) \in U$, the relation $z(t) \neq 0$ is valid for all $t \in\left[0, t_{g}\right)$, where $z(t)$ is the solution of the Cauchy problem (2.8). 
Theorem 2. (a) Let $\alpha>\beta$. Then the strategy (4.1) guarantees escaping on the time interval $\left[0, t_{g}\right)$ in the game $(U, V)$, where $t_{g}$ is the guaranteed pursuit time (see Theorem 1$)$.

(b) Let $\alpha \leq \beta$. Then the strategy (4.1) guarantees escaping on the time interval $[0,+\infty)$ in the game $(U, V)$, and the distance between the players is estimated as follows:

$$
|z(t)| \geq \begin{cases}\left|z_{0}\right|, & \text { if } \quad \alpha=\beta, \\ \left|z_{0}\right|-\frac{(\alpha-\beta) t^{2}}{2}, & \text { if } \quad \alpha<\beta .\end{cases}
$$

P r o o f. (a) Assume that $\alpha>\beta$ and the pursuer picks a control $u(\cdot) \in U$ while the evader applies the strategy (4.1). In accordance with (3.1), it follows the function

$$
z(t)=z_{0}+\int_{0}^{t}(t-s) u(s) d s+\int_{0}^{t}(t-s) \beta \xi_{0} d s .
$$

Estimate the absolute value of (4.2) as follows:

$$
\begin{aligned}
& |z(t)| \geq\left|z_{0}+\int_{0}^{t}(t-s) \beta \xi_{0} d s\right|-\left|\int_{0}^{t}(t-s) u(s) d s\right| \geq \\
& \geq\left|z_{0}\right|\left(1-\frac{\beta t^{2}}{2\left|z_{0}\right|}\right)-\int_{0}^{t}(t-s) \alpha d s=\left|z_{0}\right|-\frac{t^{2}}{2}(\alpha-\beta) .
\end{aligned}
$$

Relying on Theorem 1, we can write the estimation

$$
|z(t)| \geq\left|z_{0}\right|-\frac{(\alpha-\beta) t^{2}}{2}>0
$$

for all $t, 0 \leq t<t_{g}$.

(b) Suppose that $\alpha \leq \beta$. In this case, a proof is similar to the proof of item (a), i. e.,

$$
|z(t)| \geq\left|z_{0}\right|-\frac{(\alpha-\beta) t^{2}}{2}>\left|z_{0}\right|>0
$$

for all $t \in[0,+\infty)$. Theorem 2 is proved.

\section{An attainability domain of the pursuer}

In accordance with Theorem 1, if $\alpha>\beta$ then, by the $\Pi$-strategy (3.2), the evader is captured at some point in the space $\mathbb{R}^{n}$. In the considered game, we will construct a set of meeting points of the players for the case $\alpha>\beta$.

Let a triplet $\left(y_{0}, y_{1}, v(\cdot)\right), v(\cdot) \in V$, generates a trajectory of an evader $E$ in the form (2.6) while a triplet $\left(x_{0}, x_{1}, \mathbf{u}\left(z_{0}, v(\cdot)\right), \mathbf{u}\left(z_{0}, v(\cdot)\right) \in U\right.$, generates a trajectory of a pursuer $P$ in the form

$$
x(t)=x_{0}+x_{1} t+\int_{0}^{t}(t-s) \mathbf{u}\left(z_{0}, v(s)\right) d s,
$$

where $t \in\left[0, t^{*}\right], 0<t^{*} \leq t_{g}$, and $t^{*}$ is the encounter time of the players, that is, the equality $x\left(t^{*}\right)=y\left(t^{*}\right)$ holds. Thus, for each pair $(x(t), y(t))$, we form the set

$$
W(t)=W(x(t), y(t))=\{\omega:|\omega-x(t)| \geq(\alpha / \beta)|\omega-y(t)|\},
$$


on $\left[0, t^{*}\right]$. Note that

$$
W(0)=W\left(x_{0}, y_{0}\right)=\left\{\omega:\left|\omega-x_{0}\right| \geq(\alpha / \beta)\left|\omega-y_{0}\right|\right\} .
$$

Since $|z(t)| \geq 0$ on $\left[0, t^{*}\right]$, it is obvious that the inclusion $y(t) \in W(t)$ is valid on this time interval.

Remark 1. Note that the trajectories $x(t)$ and $y(t)$ of the players and the multi-valued mapping $W(t)$ directly depend on the choice of a control $v(\cdot) \in V$. This dependence is omitted for brevity.

Lemma 4. The multi-valued mapping $W(t)$ can be expressed as

$$
W(t)=x(t)+\Lambda(t, v(\cdot))\left[W(0)-x_{0}\right]
$$

where $\Lambda(t, v(\cdot))$ is the convergence function of the form (3.4) and

$$
W(0)=x_{0}-c\left(z_{0}\right)+R\left(z_{0}\right) S, \quad c\left(z_{0}\right)=\frac{\alpha^{2} z_{0}}{\alpha^{2}-\beta^{2}}, \quad R\left(z_{0}\right)=\frac{\alpha \beta\left|z_{0}\right|}{\alpha^{2}-\beta^{2}},
$$

and $S$ is the unit ball centered at the origin of the space $\mathbb{R}^{n}$.

P r o o f. First, write the set (5.2) as follows:

$$
W(t)=x(t)+\{\omega:|\omega| \geq(\alpha / \beta)|\omega+z(t)|\}=x(t)-c(z(t))+R(z(t)) S,
$$

where

$$
c(z(t))=\frac{\alpha^{2} z(t)}{\alpha^{2}-\beta^{2}}, \quad R(z(t))=\frac{\alpha \beta|z(t)|}{\alpha^{2}-\beta^{2}} .
$$

Now, by (3.6) and (5.4), the functions $c(z(t))$ and $R(z(t))$ can be written in the form

$$
c(z(t))=c\left(z_{0}\right) \Lambda(t, v(\cdot)), \quad R(z(t))=R\left(z_{0}\right) \Lambda(t, v(\cdot)) .
$$

Hence, we derive the validity of formula (5.3).

Corollary 1. Lemma 4 implies that, for each $t \in\left[0, t^{*}\right]$, the multi-valued mapping $W(t)$ is a ball of radius $R\left(z_{0}\right) \Lambda(t, v(\cdot))$ centered at the point $x(t)-c\left(z_{0}\right) \Lambda(t, v(\cdot))$ and the set $W(0)$ is a ball of the radius $R\left(z_{0}\right)$ centered at the point $x(0)-c\left(z_{0}\right)$.

Lemma 5 (The main lemma). The multi-valued mapping $W(t)-t x_{1}$ is monotone decreasing in $t \in\left[0, t^{*}\right]$ with respect to embedding, i.e., if $t_{1}, t_{2} \in\left[0, t^{*}\right]$ and $t_{1}<t_{2}$, then

$$
W\left(t_{2}\right)-t_{2} x_{1} \subset W\left(t_{1}\right)-t_{1} x_{1}
$$

P r o o f. From the statement of the problem, we have geometric constraint of the form (2.4) on values of the evader's acceleration vector. As a consequence, we find that

$$
|v(t)|^{2} \leq \frac{\beta^{2}}{\alpha^{2}-\beta^{2}}\left(\alpha^{2}-|v(t)|^{2}\right) .
$$

From the form of the resolving function (3.3), it is easy to ensure in the validity of the equality

$$
\alpha^{2}-|v(t)|^{2}=\lambda\left(z_{0}, v(t)\right)\left(\lambda\left(z_{0}, v(t)\right)-2\left\langle v(t), \xi_{0}\right\rangle\right) .
$$


Due to this, inequality (5.5) takes the form

$$
|v(t)|^{2}+\frac{2 \beta^{2}}{\alpha^{2}-\beta^{2}}\left\langle v(t), \xi_{0}\right\rangle \lambda\left(z_{0}, v(t)\right) \leq \frac{\beta^{2}}{\alpha^{2}-\beta^{2}} \lambda^{2}\left(z_{0}, v(t)\right) .
$$

Completing the square in this inequality, we obtain the following inequality:

$$
\left|v(t)+\frac{\beta^{2}}{\alpha^{2}-\beta^{2}} \lambda\left(z_{0}, v(t)\right) \xi_{0}\right| \leq \frac{\alpha \beta}{\alpha^{2}-\beta^{2}} \lambda\left(z_{0}, v(t)\right) .
$$

However, for all $\psi \in \mathbb{R}^{n},|\psi|=1$, the relation

$$
\left\langle v(t)+\frac{\beta^{2}}{\alpha^{2}-\beta^{2}} \lambda\left(z_{0}, v(t)\right) \xi_{0}, \psi\right\rangle \leq\left|v(t)+\frac{\beta^{2}}{\alpha^{2}-\beta^{2}} \lambda\left(z_{0}, v(t)\right) \xi_{0}\right|
$$

holds. Then, using the inequality (5.6), we find that

$$
\left\langle v(t)+\frac{\beta^{2}}{\alpha^{2}-\beta^{2}} \lambda\left(z_{0}, v(t)\right) \xi_{0}, \psi\right\rangle \leq \frac{\alpha \beta}{\alpha^{2}-\beta^{2}} \lambda\left(z_{0}, v(t)\right) .
$$

Integrate both sides of this inequality over the interval $[0, t]$ :

$$
\int_{0}^{t}\left\langle v(s)+\frac{\beta^{2}}{\alpha^{2}-\beta^{2}} \lambda\left(z_{0}, v(s)\right) \xi_{0}, \psi\right\rangle d s \leq \frac{\alpha \beta}{\alpha^{2}-\beta^{2}} \int_{0}^{t} \lambda\left(z_{0}, v(s)\right) d s .
$$

For the left-hand side of (5.7), we can write the following equalities:

$$
\begin{aligned}
\int_{0}^{t}\langle v(s)+ & \left.\frac{\beta^{2}}{\alpha^{2}-\beta^{2}} \lambda\left(z_{0}, v(s)\right) \xi_{0}, \psi\right\rangle d s=\int_{0}^{t}\left\langle v(s)+\left(\frac{\alpha^{2}}{\alpha^{2}-\beta^{2}}-1\right) \lambda\left(z_{0}, v(s)\right) \xi_{0}, \psi\right\rangle d s= \\
& =\int_{0}^{t}\left\langle v(s)-\lambda\left(z_{0}, v(s)\right) \xi_{0}, \psi\right\rangle d s+\left\langle\frac{\alpha^{2}}{\alpha^{2}-\beta^{2}} \xi_{0}, \psi\right\rangle \int_{0}^{t} \lambda\left(z_{0}, v(s)\right) d s .
\end{aligned}
$$

By the definition of $\Pi$-strategy (3.2) and in view of the form of the vector $c\left(z_{0}\right)$ in $(5.4)$, the latter equality takes the form

$$
\begin{gathered}
\int_{0}^{t}\left\langle v(s)+\frac{\beta^{2}}{\alpha^{2}-\beta^{2}} \lambda\left(z_{0}, v(s)\right) \xi_{0}, \psi\right\rangle d s=\left\langle\int_{0}^{t} \mathbf{u}\left(z_{0}, v(s)\right) d s, \psi\right\rangle+ \\
+\frac{\left\langle c\left(z_{0}\right), \psi\right\rangle}{\left|z_{0}\right|} \int_{0}^{t} \lambda\left(z_{0}, v(s)\right) d s .
\end{gathered}
$$

Taking into account the form of $R\left(z_{0}\right)$ in (5.4), for the right-hand side of inequality (5.7), we obtain

$$
\frac{\alpha \beta}{\alpha^{2}-\beta^{2}} \int_{0}^{t} \lambda\left(z_{0}, v(s)\right) d s=\frac{R\left(z_{0}\right)}{\left|z_{0}\right|} \int_{0}^{t} \lambda\left(z_{0}, v(s)\right) d s .
$$

Thus, by (5.7), (5.8), and (5.9), we have the relation

$$
\left\langle\int_{0}^{t} \mathbf{u}\left(z_{0}, v(s)\right) d s, \psi\right\rangle+\frac{\left\langle c\left(z_{0}\right), \psi\right\rangle-R\left(z_{0}\right)}{\left|z_{0}\right|} \int_{0}^{t} \lambda\left(z_{0}, v(s)\right) d s \leq 0 .
$$


Applying the properties of the support function [7]

$$
F(W, \psi)=\sup _{\omega \in W}\langle\omega, \psi\rangle
$$

in $\psi \in \mathbb{R}^{n},|\psi|=1$, and using formulas (3.4), (5.1), (5.3), and (5.4) we can find the derivative of $W(t)$ in $t$ as follows:

$$
\begin{gathered}
\frac{d}{d t} F(W(t), \psi)=\frac{d}{d t} F\left(x_{0}+x_{1} t+\int_{0}^{t}(t-s) \mathbf{u}\left(z_{0}, v(s)\right) d s+\Lambda(t, v(\cdot))\left[R\left(z_{0}\right) S-c\left(z_{0}\right)\right], \psi\right)= \\
=\left\langle x_{1}, \psi\right\rangle+\left\langle\int_{0}^{t} \mathbf{u}\left(z_{0}, v(s)\right) d s, \psi\right\rangle+\frac{\left\langle c\left(z_{0}\right), \psi\right\rangle-R\left(z_{0}\right)}{\left|z_{0}\right|} \int_{0}^{t} \lambda\left(z_{0}, v(s)\right) d s .
\end{gathered}
$$

From this and inequality (5.10), we get $\frac{d}{d t} F\left(W(t)-t x_{1}, \psi\right) \leq 0$ for all $\psi \in \mathbb{R}^{n},|\psi|=1$. This completes the proof of Lemma 5.

Corollary 2. Lemma 5 implies the inclusion $W(t) \subset W(0)+t x_{1}$ for all $t \in\left[0, t^{*}\right]$.

By Lemma 5, we obtain an attainability domain of the evader.

Lemma 6. The inclusion

$$
y(t) \in W(0)+t x_{1}
$$

holds for all $t \in\left[0, t^{*}\right]$.

$\mathrm{P} \mathrm{r}$ o o f. The inclusion (5.11) easily follows from the form of the multi-valued mapping (5.2) and Corollary 2.

Corollary 3. Lemma 6 implies that, if the initial velocities of the players are equal to zero, i.e., $x_{1}=y_{1}=0$, then the boundary of the attainability set of the evader is the Apollonius sphere of the form (5.4) in $\mathbb{R}^{n}$.

To solve the "Lifeline" game in favor of the pursuer, using (5.11), one can define the set

$$
W_{P}=\bigcup_{t=0}^{t_{g}}\left\{W(0)+t x_{1}\right\},
$$

which is obviously convex and closed. Here

$$
t_{g}=\sqrt{2\left|z_{0}\right| /(\alpha-\beta)}
$$

(see Theorem 1). We call the set $W_{P}$ the attainability domain of the pursuer.

\section{A solution of the "Lifeline" game}

In this section, the "Lifeline" game will be considered only in the case $\alpha>\beta$ and $x_{1}=y_{1}$. Hereinafter, we will omit these conditions in statements for brevity.

Definition 11. We say that the $\Pi$-strategy (3.2) provides winning for the pursuer in the "Lifeline" game on the time interval $\left[0, t_{g}\right]$ if there exists a time $t^{*} \in\left[0, t_{g}\right]$ such that the following conditions are valid: 
(a) $x\left(t^{*}\right)=y\left(t^{*}\right)$

(b) $y(s) \notin M$ for all $s \in\left[0, t^{*}\right]$.

Theorem 3. If the attainability domain $W_{P}$ of the pursuer does not intersect the set $M$, i.e., $W_{P} \cap M=\emptyset$ in the "Lifeline" game, then the $\Pi$-strategy (3.2) provides winning for the pursuer $P$.

P r o o f follows immediately from Theorem 1, Lemma 5, and Lemma 6.

Now let us consider a solution of the "Lifeline" game in favor of the evader $E$.

Definition 12. It is said that a control $v^{*}(\cdot) \in V$ provides winning for the evader in the "Lifeline" game if, for any control of the pursuer $u(\cdot) \in U$, at least one of the following conditions holds:

a) there exists a finite time $\tau$ satisfying the inclusion $y(\tau) \in M$ and the relation $x(t) \neq y(t)$ for all $t \in[0, \tau)$;

b) $x(t) \neq y(t)$ for all $t \geq 0$.

Consider the set

$$
W_{E}=\left\{\omega^{*}: \omega^{*}=\sqrt{2\left|\omega-y_{0}\right| / \beta} x_{1}+\omega, \omega \in W(0)\right\} .
$$

We call the set $W_{E}$ the attainability domain of the evader.

Theorem 4. If the attainability domain $W_{E}$ of the evader intersects the set $M$, i.e., $W_{P} \cap M \neq \emptyset$ in the "Lifeline" game, then there exists a control $v^{*}(\cdot) \in V$ that provides winning for the evader $E$.

P r o o f. Depending on the theorem conditions, there exists a point $\omega^{*} \in W_{E} \cap M$ such that the following equality holds:

$$
\omega^{*}=\sqrt{2\left|\omega-y_{0}\right| / \beta} x_{1}+\omega, \quad \omega \in W(0) .
$$

Then we prescribe the constant control of the form

$$
v^{*}=\beta\left(\omega-y_{0}\right) /\left|\omega-y_{0}\right|
$$

for the evader $E$.

First of all, let us show that, by means of the control (6.1), the evader $E$ reaches the chosen point $w^{*}$ at the time $\tau=\sqrt{2\left|\omega-y_{0}\right| / \beta}$. To this end, substituting (6.1) into (2.6), we form

$$
y(\tau)=y_{0}+y_{1} \tau+\int_{0}^{\tau}(\tau-s) v^{*} d s=y_{0}+y_{1} \tau+\frac{\tau^{2}}{2} v^{*} .
$$

From (6.1) and (6.2) and taking into account the equality $x_{1}=y_{1}$, we obtain

$$
y(\tau)=y_{0}+x_{1} \sqrt{2\left|\omega-y_{0}\right| / \beta}+\omega-y_{0}=\omega^{*} .
$$

Now let us prove that condition $(a)$ of Definition 12 holds, that is, the evader remains uncaught. Suppose the opposite, i.e., that there is some control $u^{*}(\cdot) \in U$ of the pursuer, the implementation 
of which satisfies the equality $x(t)=y(t)$ at time $\tilde{t}$ less than $\tau$, i.e., $\tilde{t}<\tau$. Then, by equation (3.1), we can write

$$
z(\tilde{t})=z_{0}+\int_{0}^{\tilde{t}}(\tilde{t}-s)\left(u^{*}(s)-v^{*}\right) d s=0 .
$$

Thus, for the right-hand side of $z(\tilde{t})$, we can write the absolute estimations:

$$
\left|z_{0}-\int_{0}^{\tilde{t}}(\tilde{t}-s) v^{*} d s\right| \leq \int_{0}^{\tilde{t}}(\tilde{t}-s)\left|u^{*}(s)\right| d s \leq \alpha \frac{\tilde{t}^{2}}{2}
$$

or

$$
\left|z_{0}-\frac{\tilde{t}^{2}}{2} v^{*}\right| \leq \alpha \frac{\tilde{t}^{2}}{2}
$$

Introducing the notation $\eta=\tilde{t}^{2} / 2$ in (6.3) and taking into account that $\left|v^{*}\right|=\beta$, we obtain the quadratic inequality in terms of $\eta$ in the form

$$
\left(\alpha^{2}-\beta^{2}\right) \eta^{2}+2 \eta\left\langle z_{0}, v^{*}\right\rangle-\left|z_{0}\right|^{2} \geq 0
$$

It follows that

$$
\eta=\frac{\tilde{t}^{2}}{2} \geq \frac{1}{\alpha^{2}-\beta^{2}}\left(\sqrt{\left\langle z_{0}, v^{*}\right\rangle^{2}+\left(\alpha^{2}-\beta^{2}\right)\left|z_{0}\right|^{2}}-\left\langle z_{0}, v^{*}\right\rangle\right) .
$$

By the assumption

$$
\frac{\tau^{2}}{2}=\left|\omega-y_{0}\right| / \beta>\frac{\tilde{t}^{2}}{2}
$$

and (6.4), we determine the relation

$$
\frac{\left|w-y_{0}\right|}{\beta}>\frac{1}{\alpha^{2}-\beta^{2}}\left(\sqrt{\left\langle z_{0}, v^{*}\right\rangle^{2}+\left(\alpha^{2}-\beta^{2}\right)\left|z_{0}\right|^{2}}-\left\langle z_{0}, v^{*}\right\rangle\right) .
$$

According to the control of the evader (6.1) and by inequality (6.5), we get

$$
\frac{\alpha}{\beta}\left|w-y_{0}\right|>\left|w-x_{0}\right|,
$$

i.e., the inclusion $\omega \in W(0)$ (see (5.2)) does not hold, which contradicts our assumption. Theorem 4 is proved.

Remark 2. Using the definitions of the attainability domain $W_{P}$ of the pursuer and the attainability domain $W_{E}$ of the evader, it is not difficult to ensure that the inclusion $W_{E} \subset W_{P}$ is valid in the "Lifeline" game.

\section{Examples}

Example 1. (Problem for the case with a lifeline). Let the game (2.1)-(2.4) be given as follows:

$$
\begin{gathered}
\ddot{x}=u, \quad x_{0}=(0,0), \quad x_{1}=(0,1), \quad|u(t)| \leq \sqrt{2}, \quad t \geq 0, \\
\ddot{y}=v, \quad y_{0}=(0,-1), \quad y_{1}=(0,1), \quad|v(t)| \leq 1, \quad t \geq 0 .
\end{gathered}
$$

Then, according to Theorem 1 , we have $t_{g}=\sqrt{2 \sqrt{2}+2}$. By Lemma 4, we can write the set

$$
W(0)=\{\omega:|\omega-c| \leq R, c=(0,-2), R=\sqrt{2}\} .
$$




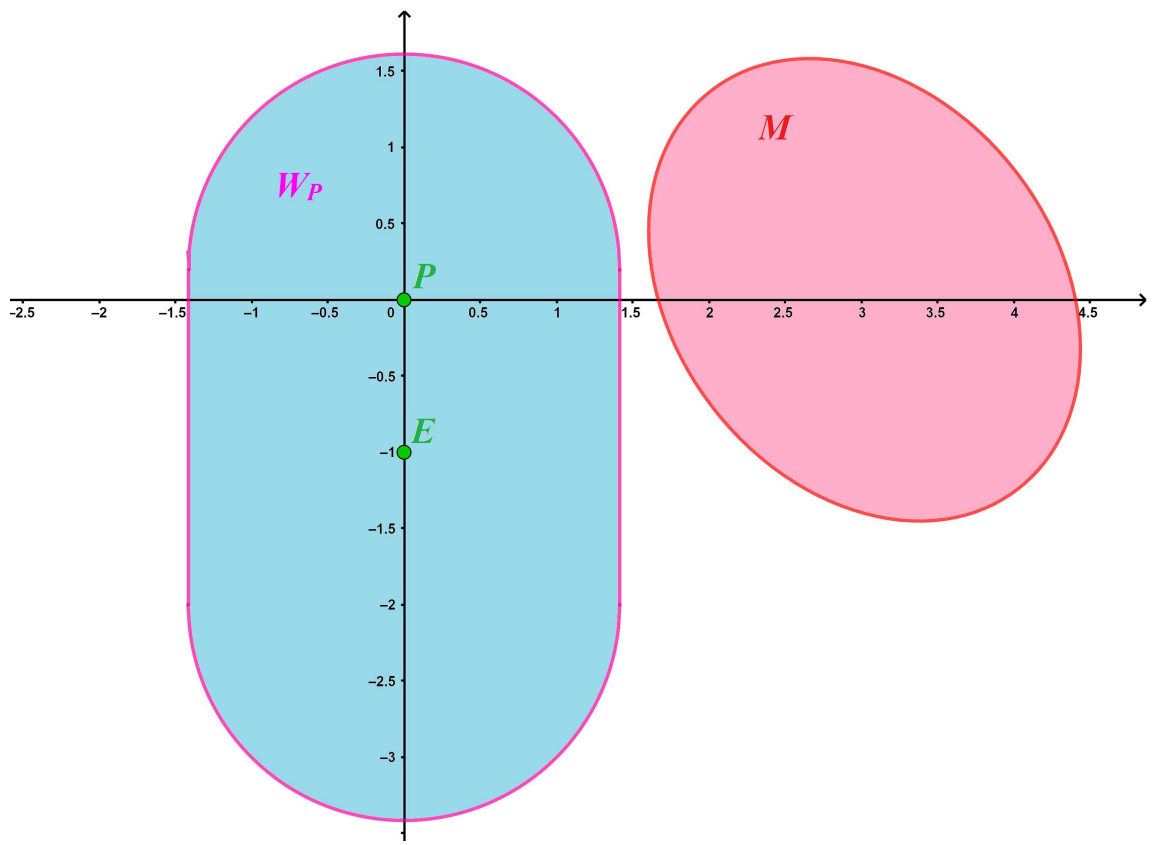

Figure 1. The case where the pursuer $P$ wins in the "Lifeline" game (7.1)-(7.2).

The boundary of $W(0)$ is

$$
\partial W(0)=\left\{\hat{\omega}=\left(\hat{\omega}_{1}, \hat{\omega}_{2}\right): \hat{\omega}_{1}^{2}+\left(\hat{\omega}_{2}+2\right)^{2}=2\right\},
$$

and the following equality is valid for these points:

$$
\left|\hat{\omega}-y_{0}\right|=\sqrt{\hat{\omega}_{1}^{2}+\left(\hat{\omega}_{2}+1\right)^{2}} .
$$

By (7.3) and (7.4), we obtain the set

$$
W_{E}=\left\{\omega^{*}=\left(\tilde{\omega}_{1}, \tilde{\omega}_{2}\right): \tilde{\omega}_{2}=\sqrt{2 \sqrt{3 \pm 2 \sqrt{2-\tilde{\omega}_{1}^{2}}}} \pm \sqrt{2-\tilde{\omega}_{1}^{2}}-2\right\} .
$$

Fig. 1 and 2 show the shapes of the sets $W_{P}$ and $W_{E}$ in the "Lifeline" game (7.1)-(7.2).

Example 2. (Attainability domain in the case of many pursuers and one evader). Consider the following game example:

$$
\begin{gathered}
\ddot{x}_{i}=u_{i}, \quad x_{i}(0)=x_{i 0}, \quad \dot{x}_{i}(0)=\eta, \quad\left|u_{i}(t)\right| \leq \mu_{i}, \quad t \geq 0 \\
\ddot{y}=v, \quad y(0)=y_{0}, \quad \dot{y}(0)=\eta, \quad|v(t)| \leq 1, \quad t \geq 0
\end{gathered}
$$

where $\mu_{i}>1$ and $x_{i 0} \neq y_{0}, i=\overline{1, m}$.

By Lemma 6, we have

$$
y(t) \in \bigcap_{i=1}^{m} W_{i 0}+t \eta
$$

where

$$
W_{i 0}=x_{i 0}-c_{i 0}+R_{i 0} S, \quad c_{i 0}=\frac{\mu_{i}^{2}}{\mu_{i}^{2}-1} z_{i 0}, \quad R_{i 0}=\frac{\mu_{i}}{\mu_{i}^{2}-1}\left|z_{i 0}\right|, \quad z_{i 0}=x_{i 0}-y_{0} .
$$




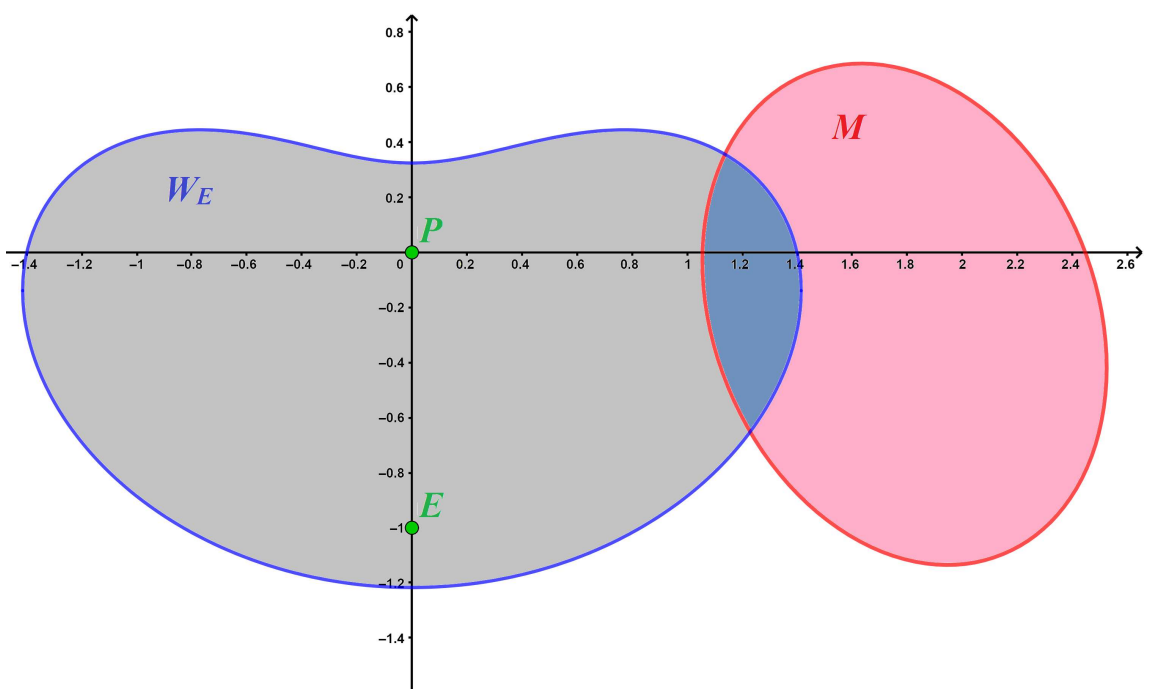

Figure 2. The case where the evader $E$ wins in the "Lifeline" game (7.1)-(7.2).

The attainability domain of the pursuers in the game (7.5)-(7.6) has the form

$$
W_{P}=\bigcup_{t=0}^{T^{*}}\left[\bigcap_{i=1}^{m} W_{i 0}+t \eta\right],
$$

where

$$
T^{*}=\min _{i=\overline{1, m}} \sqrt{\frac{2\left|z_{i 0}\right|}{\mu_{i}-1}} .
$$

\section{Conclusion}

In this paper, we have considered the pursuit-evasion problems and the "Lifeline" game of one pursuer and one evader for the inertial movements when the initial velocity vectors of the players are the same. We have imposed geometric constraints on the controls of the players. The $\Pi$-strategy was suggested for the pursuer and given optimal pursuit time in the pursuit problem. We have proposed a specific strategy for the evader. By this strategy, it was proved that the $\Pi$-strategy is optimal and the evasion is possible from the given initial states. As the main result, we have obtained the main lemma (Lemma 5) and applied this lemma to solve the "Lifeline" game.

A "Lifeline" game of many players, when geometric constraints are imposed on controls of players, can be studied in further research.

\section{REFERENCES}

1. Alekseev V.M., Tikhomirov V.M., Fomin S. V. Optimal'noye upravleniye [Optimal Control]. Moscow: Nauka, 1979. 432 p. (in Russian)

2. Aubin J.-P., Cellina A. Differential Inclusions. Set-Valued Maps and Viability Theory. Berlin-HeidelbergNew York-Tokyo: Springer-Verlag, 1984. 342 p. DOI: 10.1007/978-3-642-69512-4

3. Azamov A. On the quality problem for simple pursuit games with constraint. Serdica Bulgariacae Math. Publ., 1986. Vol. 12, No. 1, P. 38-43. (in Russian)

4. Azamov A. A., Samatov B.T. The П-strategy: analogies and applications. In: Proc. The Fourth Int. Conf. Game Theory and Management (GTM 2010), June 28-30, 2010, St. Petersburg, Russia, vol. 4. 2010. P. 33-47. 
5. Berkovitz L. D. Differential game of generalized pursuit and evasion. SIAM J. Contr., 1986. Vol. 24, No. 3. P. 361-373. DOI: 10.1137/0324021

6. Blagodatskikh A.I., Petrov N.N. Konfliktnoe vzaimodejstvie grupp upravlyaemyh ob"ektov [Conflict Interaction Between Groups of Controlled Objects]. Izhevsk: Udmurt State University, 2009. 266 p. (in Russian)

7. Blagodatskikh V.I. Vvedenie v optimal'noye upravleniye [Introduction to Optimal Control]. Moscow: Vysshaya Shkola, 2001. 239 p. (in Russian)

8. Chikrii A. A. Conflict-Controlled Processes. Dordrecht: Kluwer Academic Publishers, 1997.404 p. DOI: $10.1007 / 978-94-017-1135-7$

9. Dar'in A. N., Kurzhanskii A. B. Control under indeterminacy and double constraints. Differ. Equ., 2003. Vol. 39, No. 11. P. 1554-1567. DOI: 10.1023/B:DIEQ.0000019347.24930.a3

10. Elliot R. J., Kalton N. J. The existence of value in differential games of pursuit and evasion. J. Differential Equations, 1972. Vol. 12, No. 3. P. 504-523. DOI: 10.1016/0022-0396(72)90022-8

11. Fleming W. H. The convergence problem for differential games, II. In: Advances in Game Theory, 2nd ed. Annals of Math., vol. 52. 1964. P. 195-210. DOI: 10.1515/9781400882014-013

12. Friedman A. Differential Games, 2nd ed. Pure Appl. Math., vol. 25. New York: Wiley Int., 1971.350 p.

13. Grigorenko N. L. Matematicheskie metody upravleniya neskol'kimi dinamicheskimi processami [Mathematical Methods of Control for Several Dynamic Processes]. Moscow: Izdat. Gos. Univ., 1990. 198 p. (in Russian)

14. Hajek O. Pursuit Games: An Introduction to the Theory and Applications of Differential Games of Pursuit and Evasion. Mineola, NY: Dove. Pub. Inc., 2008. 266 p.

15. Ho Y., Bryson A., Baron S. Differential games and optimal pursuit-evasion strategies. IEEE Trans. Autom. Control, 1965. Vol. 10, No. 4. P. 385-389. DOI: 10.1109/TAC.1965.1098197

16. Ibragimov G. I. A game of optimal pursuit of one object by several. J. Appl. Math. Mech., 1998. Vol. 62, No. 2, P. 187-192. DOI: 10.1016/S0021-8928(98)00024-0

17. Ibragimov G. I. Optimal pursuit with countably many pursuers and one evader. Differ. Equ., 2005. Vol. 41, No. 5. P. 627-635. DOI: 10.1007/s10625-005-0198-y

18. Ibragimov G. I. The optimal pursuit problem reduced to an infinite system of differential equations. J. Appl. Math. Mech., 2013. Vol. 77, No. 5, P. 470-476. DOI: 10.1016/j.jappmathmech.2013.12.002

19. Ibragimov G. I. Optimal pursuit time for a differential game in the Hilbert space $l_{2}$. Sci. Asia, 2013. Vol. 39S. P. 25-30. DOI: 10.2306/scienceasia1513-1874.2013.39S.025

20. Isaacs R. Differential Games. NY: John Wiley and Sons, 1965. 385 p.

21. Ivanov R.P., Ledyayev Yu. S. Time optimality for the pursuit of several objects with simple motion in a differential game. Proc. Steklov Inst. Math., 1983. Vol. 158, P. 93-103.

22. Kornev D. V., Lukoyanov N. Yu. On a minimax control problem for a positional functional under geometric and integral constraints on control actions. Proc. Steklov Inst. Math., 2016. Vol. 293, No. Supl. 1. P. S85-S100. DOI: 10.1134/S0081543816050096

23. Krasovskii N. N., Subbotin A. I. Game-Theoretical Control Problems. NY: Springer, 2011. 517 p.

24. Munts N. V., Kumkov S.S. Numerical method for solving time-optimal differential games with lifeline. Mat. Teor. Igr. Pril., 2018. Vol. 10, No. 3. P. 48-75. (in Russian)

25. Munts N.V., Kumkov S.S. On the coincidence of the minimax solution and the value function in a time-optimal game with a lifeline. Proc. Steklov Inst. Math., 2019. Vol. 305, No. Supl. 1. P. S125-S139. DOI: 10.1134/S0081543819040138

26. Pang J.-S., Stewart D. E. Differential variational inequalities. Math. Program., 2008. Vol. 113, No. 2, Ser. A. P. 345-424. DOI: 10.1007/s10107-006-0052-x

27. Pashkov A.G., Terekhov S.D. A differential game of approach with two pursuers and one evader. J. Optim. Theory Appl., 1987. Vol. 55, No. 2. P. 303-311. DOI: 10.1007/BF00939087

28. Petrosjan L. A. Differential Games of Pursuit. Ser. Optim, vol. 2. Singapore: World Scientific Publ., 1993. 340 p. DOI: $10.1142 / 1670$

29. Pontryagin L. S. Izbrannye trudy [Selected Works]. Moscow: MAKS Press, 2004. 551 p. (in Russian)

30. Pshenichnyi B. N. Simple pursuit by several objects. Cybernet. Systems Anal., 1976. Vol. 12, No. 5. P. 484-485.

31. Pshenichnyi B. N., Chikrii A. A., Rappoport I. S. An efficient method for solving differential games with many pursuers. Dokl. Akad. Nauk SSSR, 1981. Vol. 256, No. 3. P. 530-535. (in Russian) 
32. Samatov B. T. On a pursuit-evasion problem under a linear change of the pursuer resource. Siberian Adv. Math., 2013. Vol. 23, No. 10. P. 294-302. DOI: 10.3103/S1055134413040056

33. Samatov B. T. The pursuit-evasion problem under integral-geometric constraints on pursuer controls. Autom. Remote Control, 2013. Vol. 74, No. 7. P. 1072-1081. DOI: 10.1134/S0005117913070023

34. Samatov B. T. The П-strategy in a differential game with linear control constraints. J. Appl. Math. Mech., 2014. Vol. 78, No. 3. P. 258-263. DOI: 10.1016/j.jappmathmech.2014.09.008

35. Samatov B. T. Problems of group pursuit with integral constraints on controls of the players. I. Cybernet. Systems Anal., 2013. Vol. 49, No. 5. P. 756-767. DOI: 10.1007/s10559-013-9563-7

36. Samatov B. T. Problems of group pursuit with integral constraints on controls of the players. II. Cybernet. Systems Anal., 2013. Vol. 49, No. 6. 907-921. DOI: 10.1007/s10559-013-9581-5

37. Samatov B. T., Ibragimov G., Khodjibayeva I. V. Pursuit-evasion differential games with Grönwall-type constraints on controls. Ural Math. J., 2020. Vol. 6, No. 2. P. 95-107. DOI: 10.15826/umj.2020.2.010

38. Satimov N. Yu., Rikhsiev B. B., Khamdamov A. A. On a pursuit problem for n-person linear differential and discrete games with integral constraints. Math. USSR Sb., 1983. Vol. 46, No. 4. P. $459-471$. DOI: 10.1070/SM1983v046n04ABEH002946

39. Shiyuan J., Zhihua Q. Pursuit-evasion games with multi-pursuer vs. One fast evader. In: Proc. 8th World Congress on Intelligent Control and Automation, July 7-9, 2010, Jinan, China. IEEE Xplore, 2010. P. 3184-3189. DOI: 10.1109/WCICA.2010.5553770

40. Subbotin A. I. Generalization of the main equation of differential game theory. J. Optim. Theory Appl., 1984. Vol. 43, No. 1. P. 103-133. DOI: 10.1007/BF00934749

41. Subbotin A. I., Chentsov A. G. Optimizaciya garantii v zadachah upravleniya [Optimization of Guaranteed Result in Control Problems]. M: Nauka, 1981. 288 p. (in Russian)

42. Ushakov V. N. Extremal strategies in differential games with integral constraints. J. Appl. Math. Mech., 1972. Vol. 36, No. 1. P. 12-19. DOI: 10.1016/0021-8928(72)90076-7

43. Ushakov V. N., Ershov A. A., Ushakov A. V., Kuvshinov O. A. Control system depending on a parameter. Ural Math. J., 2021. Vol. 7, No. 1. P. 120-159. DOI: 10.15826/umj.2021.1.011 